\title{
Performance Assessment of UK's Cellular Network for Vehicle to Grid Energy Trading: Opportunities for $5 \mathrm{G}$ and Beyond
}

\author{
Mehdi Zeinali \\ Inst. for Digital Communications \\ School of Engineering \\ The University of Edinburgh \\ Edinburgh, UK \\ m.zeinali@ed.ac.uk
}

\author{
I. Safak Bayram \\ Dept. of Electronic and Electrical Engineering \\ Faculty of Engineering \\ University of Strathclyde \\ Glasgow, UK \\ safak.bayram@strath.ac.uk
}

\author{
John Thompson \\ Inst. for Digital Communications \\ School of Engineering \\ The University of Edinburgh \\ Edinburgh, UK \\ john.thompson@ed.ac.uk
}

\begin{abstract}
The proliferation of plug-in electric vehicles (PEV) and advances in high-speed low latency communication networks redefine the relationships between electricity providers and endusers. A group of PEV owners, coordinated by an aggregator, can participate in ancillary energy markets to stabilize electricity grids and, in return, receive payments for services rendered. However, PEVs are typically parked across a dispersed region possibly with diverse signal strength and data rates. Vehicleto-Grid (V2G) applications have tight latency (e.g., $500 \mathrm{~ms}$ to 2 seconds) and packet-loss requirements, hence, the supporting communication infrastructure should be carefully evaluated for real-world implementations. In this paper, we assess the performance of the internet-based 4G cellular network in the United Kingdom to evaluate these key metrics. We develop a low cost and easy deployable testbed platform to collect and analyze the latency and packet loss rate of different package sizes, transport protocols, and signal strengths. Due to availability of hardware resources and city-wide coverage of 4G networks, a single parking lot to aggregator scenario is emulated. The results show that in most cases current 4G network can deliver packets less than $500 \mathrm{~ms}$ which is required in fast frequency response applications in the UK. On the other hand, for more complex scenarios such as multi-aggregator to distributed clients, there is a need to use 5G and beyond to meet the latency requirements. To the best of authors' knowledge, this is the first study focusing on the field testing and assessment of an actual internet-based communication network for V2G applications.
\end{abstract}

Index Terms - vehice-to-grid, smart grid, energy markets, wireless networks, performance assessment, low cost test-bed

\section{INTRODUCTION}

Plug-in electric vehicles (PEVs) have emerged as an attractive and sustainable solution to decrease carbon emissions and energy consumption in transportation networks. In line with the United Nations' 2030 Agenda for Sustainable Development, many nations around the world have introduced policies to limit the use of combustion engine vehicles and boost PEV adoption [1]. According to recent industry forecasts [2], PEV

This work was supported by the UK Department for Business, Energy and Industrial Strategy (BEIS) project tender 1273/01/2017-ND114 (Project Quickturn). Also We thank the University of Edinburgh's Community Makerspace (UCreatStudio) which provided free to use equipment as part of our Test-Bed. sales are expected to grow from 2.4 million in 2018 to 13.2 million in 2024, which would represent $15 \%$ of total sales. In parallel, the electric power grids which are the backbone infrastructure supporting PEVs, have been transforming the way the electricity is provisioned and consumed. To host more disruptive technologies such as green renewable generators, storage units, and PEVs, communication and sensing technologies are being integrated to ensure operational continuity [3].

In power grid operations, ancillary services are critical as market operators are responsible for matching the supply with the demand in real-time within tight tolerance bounds. Traditionally, dedicated fast-response diesel generators are employed to respond to grid signals within seconds to minutes. In recent years, the participation of PEV batteries in the ancillary services market, also known as vehicle-to-grid (V2G), has gained popularity as the use of PEVs enhance system efficiency, while providing monetary benefits to PEV owners [4]. In a V2G application, each PEV battery is charged and discharged within a time window according to real-time automatic generation control signals [5]. However, due to the minimum power constraints of service contracts (typically ranges between 1 to $3 \mathrm{MW}$ ), only the aggregation of many vehicles can provide enough resource capacity [6]. Hence large scale PEV charging lots coordinated by aggregators inherently represent great potential for $\mathrm{V} 2 \mathrm{G}$ applications as they host large collections of stationary PEVs at a single place.

There has been a number of successfully implemented pilot studies, typically with 10-30 PEVs (see [4] and [7]). However, the implementation of large-scale V2G applications requires the necessary communications infrastructure to enable timely information dissemination. The PEV aggregator needs to exchange information on PEV departure times, battery state of charge, and accurate billing-related messages. Possible V2G scenarios and timelines are presented in Fig. 1. Aggregators and vehicle owners are further required to abide by the contracts which involve tight response times and the amount of power to be charged or discharged from the vehicles. 


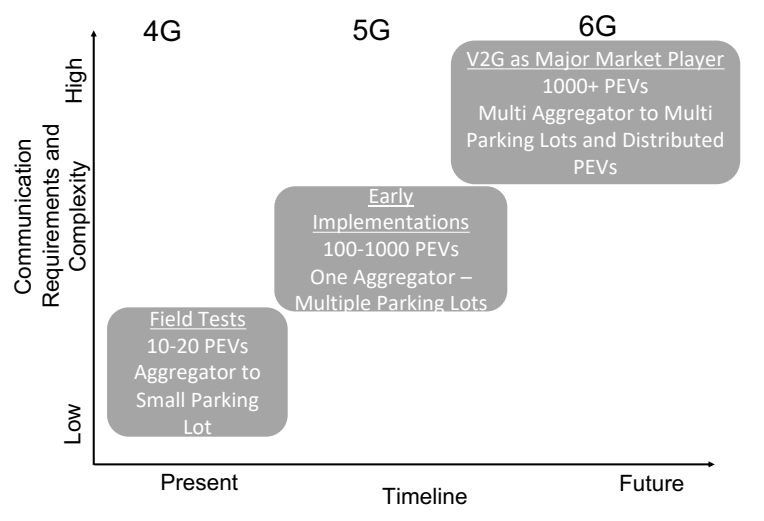

Fig. 1. V2G application size and communication requirements.

The most common ancillary services application is frequency regulation which is delay-sensitive and a number of studies showed that load frequency control is negatively affected by communication delays as PEVs cannot meet their contracted power capacity [8]-[10]. In the United Kingdom, once the market signal is sent, the response must be within $500 \mathrm{~ms}$ [10], while this is typically 2 seconds in the United States [11]. Similar to EV batteries, other demand-side applications can be used in frequency control applications. Reference [12] developed a decentralized control scheme that allows the aggregation of refrigerators. In this case communication requirement would be tighter to coordinate distributed smaller loads.

Over the last decade, there has been a growing body of literature on V2G applications for market participation and bidding strategy, charging optimization, and communication and security of aspects. One of the earliest works is presented in reference [13] in which authors investigated the reliability and availability of PEVs as ancillary services providers with and without the presence of aggregators. In an actual implementation, [7] presents an optimal bidding strategy in California's ancillary services market for a group of 30 PEVs. Coordinated bidding is further studied in references [14] and [15], however, in most studies a perfect communication system is assumed. The work presented in [9] assumes an imperfect communication network and quantifies the impacts of jitter delay in ancillary services. The authors assume that PEVs parked at a charging station communicates with power line communication, while the communication between the aggregator and the EVs are performed with the fourth generation $(4 \mathrm{G})$ wireless network. They used network simulator 3 to evaluate communication system performance. In [11], the authors investigated the sensitivity of load frequency control services in respect to wireless communication delays. They showed that long delays can cause grid instabilities.

Furthermore, there have been efforts towards the standardization of communication protocols. The Society of Automotive Engineers (SAE) has defined the J28XX and J29XX standards to define messages between PEVs and charging equipment [16]. Moreover, the Electric Power Research In-

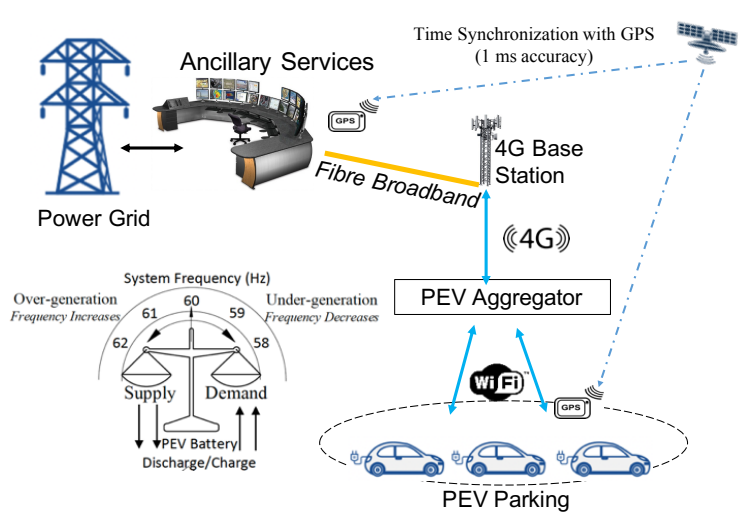

Fig. 2. Overview of the ancillary services and communication system.

stitute is working with various system operators and car manufacturers to develop a cloud-based software platform called the Open Vehicle-Grid Integration Platform for PEV communications [17]. Despite the research efforts presented above, the literature lacks studies on the measurement, testing, and performance evaluation of actual communication networks. This is particularly critical for $\mathrm{V} 2 \mathrm{G}$ applications as previous research suggests that simulation results can significantly differ from real-world implementations [18]. To that end, the main contributions of this paper are (1) the development of a hardware and software platform to evaluate the performance of an actual 4G communication network located in the United Kingdom; (2) assessment of transmission control protocol (TCP) and user datagram protocol (UDP) for V2G applications with highly accurate GPS-based time synchronization; and (3) the analysis of end-to-end latency and packet losses for varying packet sizes and wireless signal levels. To the best of authors' knowledge, this is the first study focusing on the assessment of an actual internet-based communication network for $\mathrm{V} 2 \mathrm{G}$ applications.

\section{SYSTEM OVERVIEW}

\section{A. Communication Infrastructure for $V 2 G$}

As discussed in the previous section, V2G applications have recently been rolled out and there is no standard communication infrastructure. Nevertheless, we follow the published literature (see references [9] and [13]) for possible communication architectures for real-world implementation. As depicted in Fig. 2, we consider a group of PEVs parked at charging lots such as those located at universities, hospitals, airports, or other workplaces. PEVs typically remain stationary for many hours and there is a sufficient time window to respond to market signals and charge their vehicles for the daily commute. It is assumed that each PEV can communicate with an aggregator located in the parking lot via a Wi-Fi network. Note that this communication can also be realized with power-line communication (PLC) or Zigbee [9]. Recall that the information exchange in this part is charge/discharge signals and each PEV's contribution to the V2G session. 
Next, the aggregator connects to the market operator via the $4 \mathrm{G}$ network. The aggregator will connect to the market operator first with a wireless network which is from the parking lot to the nearest base station. From the base station to the market operator, high-speed fibre connectivity is used to transmit messages. It is noteworthy that as $\mathrm{V} 2 \mathrm{G}$ applications become more common there could be more hierarchy between the aggregator(s) and the grid operator. For instance, at each parking lot, the V2G session can be coordinated via a subaggregator, while a number of parking lots can be coordinated with the main aggregator. Nevertheless, we will consider this case for future study.

It is important to note that, we differentiate latency from the operator to the PEVs and from the PEVs to the operators because latency is defined as the time to transmit a data packet across a network and could be measured in two different ways: (1) Round-trip time (RTT): the time it takes for a data packet or signal to reach a destination and come back to the source. (2) One way latency: the time it takes for sending a packet from the source to destination. In a symmetrical communication system it can be estimated that one-way latency is equal to the half of RTT. As the data flow in most smart grid applications is from the end users or devices to the grid operator and, in return, a relatively shorter control message is sent by grid operator to end users, we use one-way latency. Note also that end-points are further time synchronized by GPS receivers. Then, regardless of the transport layer protocol type, connection mechanism, or paths that data packets travel from the source to the destination, the one-way latency is defined as $t_{\text {One-way Latency }}=t_{2}-t_{1}$, where $t_{2}$ is timestamp of received data (at PEV side) and $t_{1}$ is timestamp of initiated transmission from the grid operator (or vice versa).

\section{B. Transport Layer}

Internet-based communication networks primarily use TCP or UDP protocols for various applications. It is well-known that the TCP protocol offers reliable data delivery through a three-way handshake and acknowledgment mechanisms. Lost messages are retransmitted and packets are sorted in the order they were sent. The UDP protocol, on the other hand, is considered as an unreliable protocol as it does not guarantee end-to-end packet delivery due to lack of handshake mechanism such as that used in TCP. This also means when UDP packets are dropped by a switch or a router, there will be no automatic retransmission mechanism. However, UDP is typically faster than TCP and favored in applications requiring fast data transfer. For the case of V2G applications, since there is no globally accepted transportation protocol, in this paper, we will examine the latency and packet drop performance of both protocols.

\section{Virtual Private Network}

To enable a secure communication between PEVs and the system operator, we enabled a virtual private network (VPN) connection using SoftEther software [19]. Recall that a VPN enables several remote nodes on the internet to connect to each other through encrypted communication links. It means that this private network can be deployed on top of the existing public network, making it difficult for messages to be intercepted by adversaries. In VPN tunnels, encryption is performed by encapsulating messages into a special packet format which can then be sent through the internet using conventional transport protocols. This open-source multi-protocol software enables us to connect various remote nodes (mimicking PEVs) scattered across the city of Edinburgh.

\section{GPS Synchronization}

High-resolution clock synchronization is critical for widearea communication networks. In this work, we use GPSreference timing for higher accuracy. GPS-timing estimates the time offset between the atomic clocks of GPS satellites and the receiver's internal clock via transmitting pulse per second (PPS) signals [20]. As will be discussed in the next section, we use Raspberry Pi devices to mimic the client which samples the PPS signal generated by the GPS module by using general-purpose input/output (GPIO) connections and the Linux software. This means that such applications can use the raw GPS data in conjunction with the PPS signal to obtain very accurate time measurements with a low capital cost. Provided that the GPS antenna is installed with a clear view of GPS satellites, the Raspberry Pi client can be synchronized with the satellite clock and is usable as a high precision time source. In this work, we have experimented with several GPS receivers with different specifications. Among all GPS receivers and chose the one that can remain synchronized with the satellite at $87 \%$ of the time and is capable of achieving time precision in the set-up of less than 100 microseconds.

\section{HARDWARE AND TEST-BED}

We proceed to explain the emulation set up and developed testbed. The ancillary service market operator is emulated with a laptop computer (Dell Vostro 1720) which runs a server developed in Python to receive and send messages to a client (or an aggregator). To mimic PEV actions and the time to process information exchange between PEVs and the market operator, we developed a client program on a Rasberry Pi 3B emulator. Furthermore, for secure communication, the aforementioned VPN program was loaded and run on both the client and the server. It is noteworthy that during the initial test phases, we have encountered a number of issues related to server-client connectivity. Since internet service providers periodically change IP addresses of connected devices, the communication between the client and the server is lost. One obvious solution would be to obtain static IP addresses, however, this would be a costly option. Instead, we used dynamic domain name systems (DDNS) which is a service that automatically and periodically monitors the server's IP address for changes. Whenever there is a change, a new IP address is updated on client side. However, for actual power grid applications, static IP would provide enhanced reliability in communication links. 
The server will send messages (automatic generation control) to the client based on market conditions. However, according to [21] ancillary services include various control signals. For instance, the "Regulation-up" or "Regulationdown" signal is sent for an immediate change request for the power output, while "Regulation Mileage-up/down" signals require actions within 2-4 seconds. Hence, we use message lengths from $50 \mathrm{~B}$ to $2 \mathrm{~KB}$ to imitate different scenarios. It should be noted that for most of the smart grid applications such as wide-area monitoring systems, demand response or vehicle to grid, unplanned events occur randomly, hence, such events can be counted as an isolated packet transmission scenario. To mimic this case, packets are spaced by $60 \mathrm{sec}$. Furthermore, to save energy most $4 \mathrm{G}$ modems stay in sleep mode when not transmitting. With this packet spacing, we can further measure the time it takes for $4 \mathrm{G}$ modem to change its state of sleep mode to active mode.

In real-world applications, response types may differ due to network settings. Moreover, as discussed earlier, the TCP protocol provides high reliability while UDP is faster in delivering packets. Depending on the message exchange and application, one protocol may be preferred over the other. For instance, it is expected that the UDP will present higher efficiency in transmitting short messages, provided that occasional packet losses can be managed. On the other hand, TCP may be more suitable for long messages as reliability could be a major concern. In summary, end-to-end latency depends on (1) The number of PEVs in the parking lot (Wi-Fi collisions lead to media access delays); (2) Congestion management and active queue management algorithms used in the network; (3) The number of routers and switches in the proposed network; (4) The signal strength and bandwidth of the communications links; (5) The size of transmitted data packets and reporting rate per second; and (6) Preferred transport layer protocol.

TABLE I

4G Wireless Network Signal Strength Measurements for 48 HOURS.

\begin{tabular}{cccc}
\hline \multicolumn{4}{c}{ Signal Classification [dBm] } \\
\hline Strong & Medium & Poor & Average (1-month) \\
\hline-69 & -105 & -118 & -93 \\
\hline
\end{tabular}

\section{Results And AnAlyses}

In a typical $\mathrm{V} 2 \mathrm{G}$ application, vehicles are parked in lots located in urban city centers. In such cases, signal strength is a major determinant of end-to-end delay and packet loss performance. To that end, we measure signal strength at a typical university parking site located on the university campus to mimic real-world application scenarios. As presented in Table I, 4G wireless signal strength is divided into four categories, namely, poor, medium, strong, and long-term average. In the remainder of this paper, we categorize network performance according to these signal strength levels. It is important to note that data transmission is carried out for nearly 70 hours with one-minute intervals, while the long-term signal average is based on measurements taken for one month.

In V2G applications, the most important network performance parameters are (1) end-to-end latency as it determines how fast PEVs can respond to market signals and (2) packet loss ratio as critical market signals are exchanged. We evaluate both TCP and UDP protocols as potential candidates for the transport protocol. Moreover, measurements from the client to the server are distinguished from server to client as explained in the previous section. In Figs. 3(a) and 3(b), we present the average latency for all cases. The presented latency calculations include the latency from PEV to an aggregator on a Wi-Fi link plus latency from aggregator to the grid operator server on the $4 \mathrm{G}$ link. In this calculation, the majority of the latency is associated with the $4 \mathrm{G}$ link. From our previous work [22], [23], the latency of the Wi-Fi link is up to $50 \mathrm{~ms}$ of the measured latency value. However, this value can be significantly impacted by the number of users connected to the Wi-Fi access point as a high number of users per single access point can result in congestion and thus a significant performance bottleneck. In such cases, UDP packets will be lost and will never be recovered. This can further lead to unacceptable packet delays and session disruptions. For TCP packets, congestion would increase the retransmission of packets. Since the evaluation of $\mathrm{Wi}-\mathrm{Fi}$ delay requires the deployment of hundreds of emulators, we did not implement this part and instead adopted values taken from the literature (see [24]). The results show similar latency results of 250$450 \mathrm{~ms}$ for both UDP and TCP. Next, we present the packet loss ratio for both UDP and TCP protocols. As shown in Fig. 3(c), the UDP packet loss ratio is much higher than for TCP as TCP tries to guarantee packet delivery.

Even though the results presented above provide insights about latency, holistic comparisons can only be made from cumulative distribution functions $(\mathrm{CDF})$ of latency measurements. Moreover, most service level agreements between grid operator and market participants are based on latency requirements of $90 \%$ confidence intervals or higher. To that end, we present CDF calculations for TCP and UDP for all signal levels (also presented in Table II). It can be seen from Figs. 4 and 5 that the latency value increases from $350 \mathrm{~ms}$ to $450 \mathrm{~ms}$ for $90 \%$ confidence by reducing the signal level from Strong to Poor for TCP. UDP packets can be observed to experience a higher failure rate but the trend is again similar. It is obvious from comparing Fig. 4 and Fig. 5 that for dropped data packets, there is still a chance for another transmission by UDP in the time window of a few ms up to $100 \mathrm{~ms}$ which can be considered as a fast transmission. On the other hand, transmission with TCP requires at least $100 \mathrm{~ms}$ delay which is due to the "slow start mechanism". This mechanism in TCP means that TCP is not well-suited for sending isolated control packets as it leads to higher latency. On the other hand, UDP results in lower latency, provided the packet loss rate is acceptable. Despite UDP's higher packet losses, high speed UDP communication can trigger packet retransmission within a certain time window for critical applications to enhance 


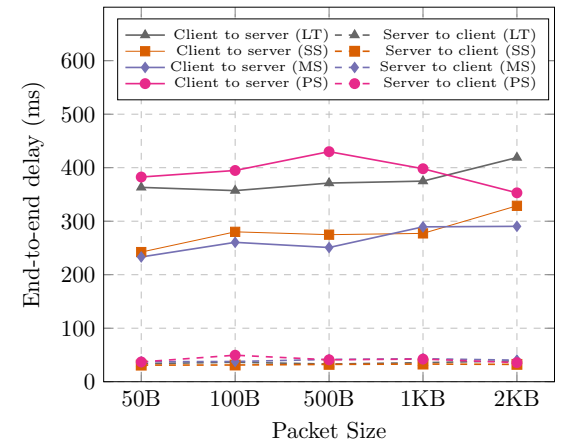

(a) TCP latency

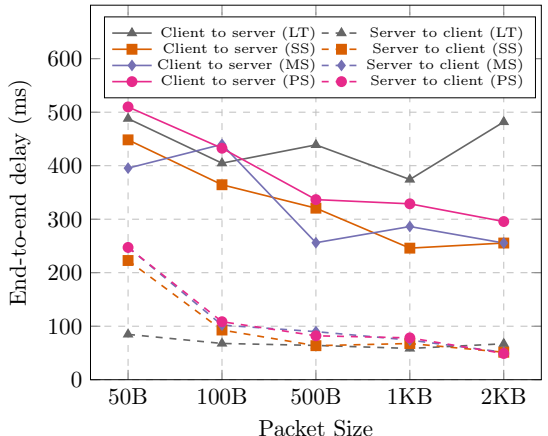

(b) UDP latency

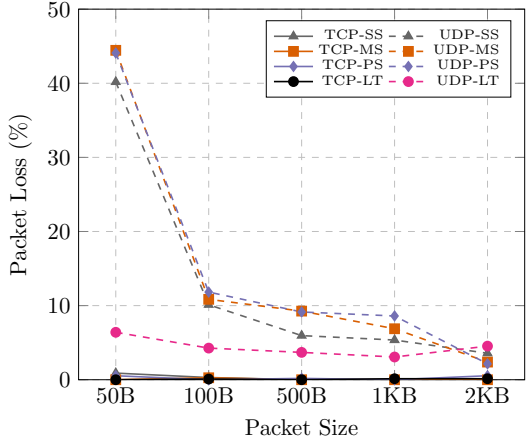

(c) Packet loss rate

Fig. 3. Network performance comparison for different signal strengths (Long-term average (LT), strong strength (SS), medium strength (MS), and poor strength (PS).

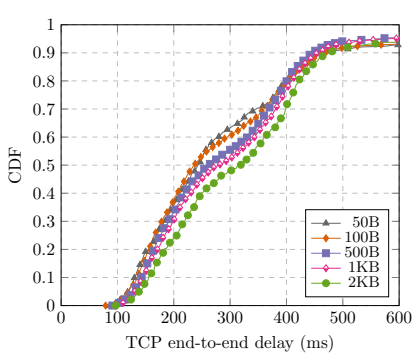

(a) Long-term average

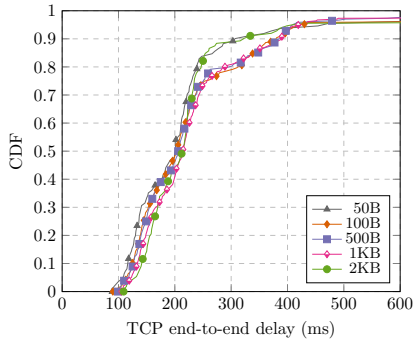

(b) Strong signal strength

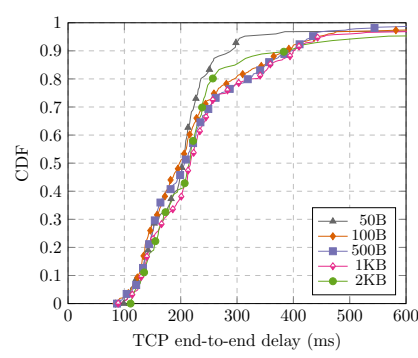

(c) Medium signal strength

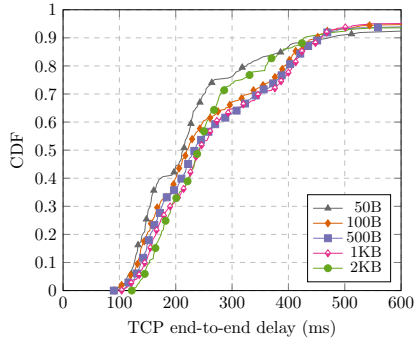

(d) Poor signal strength

Fig. 4. Cumulative distribution function (CDF) of TCP latency for varying packet sizes and signal strengths.

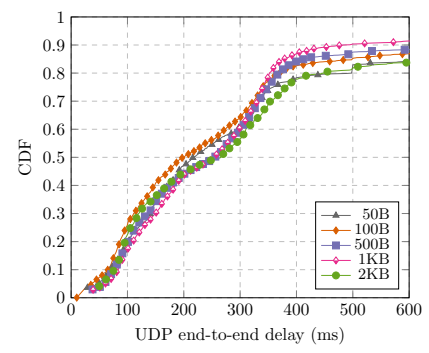

(a) Long-term average

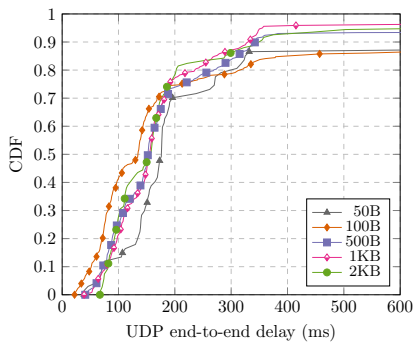

(b) Strong signal strength

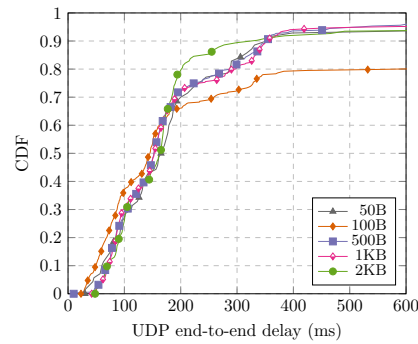

(c) Medium signal strength

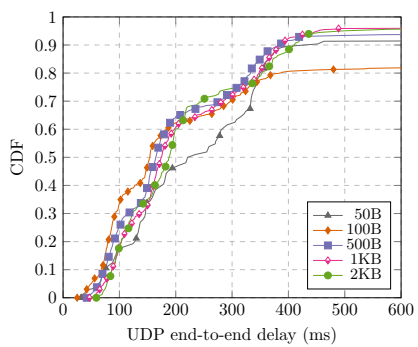

(d) Poor signal strength

Fig. 5. Cumulative distribution function (CDF) of UDP latency for varying packet sizes and signal strengths.

reliable delivery.

Moreover, previous research demonstrates that network traffic behavior can change at different days and hours of the week for a specific communication network [22]. So as a next step we will study the correlation between network congestion and V2G market signals. A possible correlation between the two parameters can provide insights for network designers. Network algorithms can be used to improve bandwidth efficiency, prioritize packets, and reduce slow-start mechanism for TCP.

In order to evaluate the effects of communication delays, system operators employ accuracy metrics. PJM is a major balancing authority in the eastern part of the United States and calculates the precision score $\left(P_{S}\right)$ with the following formula: $P_{S}=1-\frac{1}{N} \sum_{i=1}^{N}\left|\frac{I[i]-R[i]}{C}\right|$, where $N$ is the total number of market signals during a V2G session, $C$ represents the average absolute values of instructed signals, $I[i]$ is the $i^{\text {th }}$ instruction and $R[i]$ is the corresponding response. It is easy to see that, if vehicles respond to instruction within the allowed time frame such as 2 seconds as discussed in [18], then $I[i]-R[i]=0$ and precision score is equal to 1 (hundred percent precision). On the other hand, due to communication delays PEVs may receive older instructions which would make $I[i]-R[i] \neq 0$ and lead to lower precision. Then the mismatch between load and demand will be compensated by another source which will 
lead to monetary losses. Due to page limitations, we leave this case study as a future work.

TABLE II

90\% CONFIDENCE INTERVALS FOR 4G LATENCY (IN MS).

\begin{tabular}{|c|c|c|c|c|c|c|c|c|}
\hline \multirow[b]{2}{*}{ Packet } & \multicolumn{2}{|c|}{ Long $T$. } & \multicolumn{2}{|c|}{ Strong S. } & \multicolumn{2}{|c|}{ Medium S. } & \multicolumn{2}{|c|}{ Poor S. } \\
\hline & TCP & UDP & TCP & UDP & & UDP & TCP & UDP \\
\hline 50B & 468 & 1510 & 307 & 1108 & 297 & 353 & 488 & 452 \\
\hline $100 \mathrm{~B}$ & 465 & 906 & 380 & 1136 & 387 & 1425 & 452 & 1489 \\
\hline $500 \mathrm{~B}$ & 447 & 828 & 383 & 344 & 401 & 354 & 455 & 383 \\
\hline $1 \mathrm{~KB}$ & 455 & 476 & 389 & 332 & 403 & 352 & 458 & 387 \\
\hline $2 \mathrm{~KB}$ & 476 & 1408 & 350 & 350 & 600 & 418 & 450 & 419 \\
\hline
\end{tabular}

\section{Conclusions And Discussion FOR 5G AND BeyOnd}

In this paper, we evaluated the performance of the UK's internet-based communication network for vehicle to grid energy market applications. Due to city-wide coverage, we considered $4 \mathrm{G}$ and $\mathrm{WiFi}$ as wireless networks and a high-speed fibre link as the backhaul. To mimic real-world applications we measured $4 \mathrm{G}$ signal strength and categorized the findings into four groups (poor, medium, strong, and long-term average). For transport layer protocols, we considered both TCP and UDP. We calculated and discussed latency for both protocols and all signal levels. The results showed that even though UDP's overall latency performance is considerably better than TCP, high packet loss rates raise reliability issues. Moreover, by analyzing the cumulative distribution of latency, we showed that network performance provides stochastic guarantees (with a $90 \%$ confidence interval) to provide end-to-end delay below two seconds which is the case for many US energy markets, while the fast frequency response in the UK requires $500 \mathrm{~ms}$ response times.

It is important to note that the presented study considers single aggregator to a parking lot scenario and latency performance is slightly better than the market threshold in the UK. For more complicated futuristic scenarios such as multiple aggregator to multiple parking lots and distributed vehicles, then there is a need to use low latency $5 \mathrm{G}$ networks. In this case, latency statistics would differ significantly from one case to another as propagation delay would also play a critical role. However, due to lack of $5 \mathrm{G}$ roll out in cities, we leave the physical experimentation as a future work. Similarly, ultra fast low latency networks can be take part in future energy grid stabilization in which online customers who can negotiate prices to participate in a V2G session.

\section{REFERENCES}

[1] J. H. Wesseling, "Explaining variance in national electric vehicle policies," Environmental Innovation and Societal Transitions, vol. 21, pp. 28-38, 2016.

[2] $\mathrm{Lg}$ chem sees electric vehicles accounting for $15 \%$ of all car sales in 2024. [Online]. Available: https://tinyurl.com/yx3tghzq

[3] R. Schmalensee and et al., The future of solar energy: an interdisciplinary MIT study. Energy Initiative, Massachusetts Institute of Technology, May 2015.
[4] I. S. Bayram and A. Tajer, Plug-in electric vehicle grid integration. Artech House, 2017.

[5] S. Han, S. Han, and K. Sezaki, "Development of an optimal vehicle-togrid aggregator for frequency regulation," IEEE Transactions on smart grid, vol. 1, no. 1, pp. 65-72, 2010.

[6] D. M. Steward, "Critical elements of vehicle-to-grid (v2g) economics," National Renewable Energy Lab.(NREL), Golden, CO (United States), Tech. Rep., September 2017.

[7] N. DeForest, J. S. MacDonald, and D. R. Black, "Day ahead optimization of an electric vehicle fleet providing ancillary services in the los angeles air force base vehicle-to-grid demonstration," Applied energy, vol. 210, pp. 987-1001, 2018.

[8] L. Jiang, W. Yao, Q. Wu, J. Wen, and S. Cheng, "Delay-dependent stability for load frequency control with constant and time-varying delays," IEEE Transactions on Power systems, vol. 27, no. 2, pp. 932941, May 2011.

[9] K. Ko and D. K. Sung, "The effect of cellular network-based communication delays in an ev aggregator's domain on frequency regulation service," IEEE Transactions on Smart Grid, vol. 10, no. 1, pp. 65-73, Jan 2019.

[10] Q. Hong, M. Karimi, M. Sun, S. Norris, O. Bagleybter, D. Wilson, I. Abdulhadi, V. Terzija, B. Marshall, and C. Booth, "Design and validation of a wide area monitoring and control system for fast frequency response," IEEE Transactions on Smart Grid, pp. 1-11, January 2020.

[11] K. S. Ko and D. K. Sung, "The effect of ev aggregators with timevarying delays on the stability of a load frequency control system," IEEE Transactions on Power Systems, vol. 33, no. 1, pp. 669-680, January 2018.

[12] E. Vrettos, C. Ziras, and G. Andersson, "Fast and reliable primary frequency reserves from refrigerators with decentralized stochastic control," IEEE Transactions on Power Systems, vol. 32, no. 4, pp. 2924-2941, July 2016.

[13] C. Quinn, D. Zimmerle, and T. H. Bradley, "The effect of communication architecture on the availability, reliability, and economics of plug-in hybrid electric vehicle-to-grid ancillary services," Journal of Power Sources, vol. 195, no. 5, pp. 1500-1509, January 2010.

[14] M. Ansari, A. T. Al-Awami, E. Sortomme, and M. Abido, "Coordinated bidding of ancillary services for vehicle-to-grid using fuzzy optimization," IEEE Transactions on Smart Grid, vol. 6, no. 1, pp. 261-270, January 2014.

[15] J. Donadee and M. D. Ilić, "Stochastic optimization of grid to vehicle frequency regulation capacity bids," IEEE Transactions on Smart Grid, vol. 5, no. 2, pp. 1061-1069, March 2014.

[16] M. C. Falvo, D. Sbordone, I. S. Bayram, and M. Devetsikiotis, "Ev charging stations and modes: International standards," in 2014 International Symposium on Power Electronics, Electrical Drives, Automation and Motion. IEEE, 2014, pp. 1134-1139.

[17] S. Chhaya and et al. (2016, July) Open vehicle-grid integration platform: General overview. EPRI.

[18] D. Bian, M. Kuzlu, M. Pipattanasomporn, S. Rahman, and D. Shi, "Performance evaluation of communication technologies and network structure for smart grid applications," IET Communications, vol. 13, no. 8, pp. 1025-1033, May 2019.

[19] Softether vpn. [Online]. Available: https://tinyurl.com/pbmuhdj

[20] K. Koo, J. Lee, and J. Brownjohn, "Time synchronization for wireless sensors by using low-cost gps module," Frontiers in Built Environment, vol. 4, p. 82, January 2019.

[21] Z. Zhou, T. Levin, and G. Conzelmann, "Survey of us ancillary services markets," Argonne National Lab.(ANL), Argonne, IL (United States), Tech. Rep., January 2016.

[22] M. Zeinali and J. Thompson, "Implementation of highly accurate testbed for practical evaluation of wired and wireless internet based smart grid communications," in The 4th International Conference on UKChina Emerging Technologies. IEEE, 2019, pp. 1-4.

[23] M. Zeinali and J. S. Thompson, "Practical evaluation of uk internet network characteristics for demand-side response applications," in 2018 IEEE International Conference on Communications, Control, and Computing Technologies for Smart Grids (SmartGridComm). IEEE, 2018, pp. 1-6.

[24] I. Grigorik, High Performance Browser Networking: What every web developer should know about networking and web performance. O’Reilly Media, Inc.”, May 2013. 